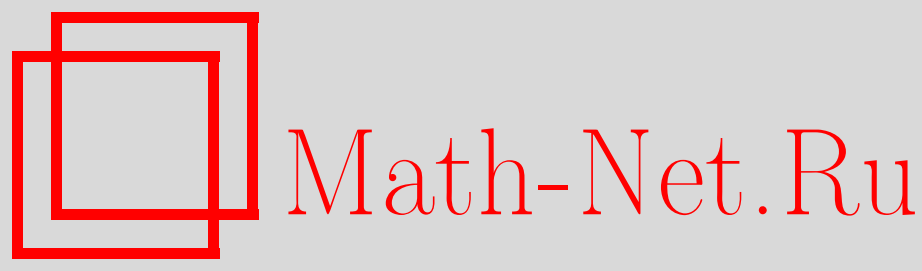

Д. П. Новиков, Б. И. Сулейманов, "Квантования" изомонодромной гамильтоновой системы Гарнье с двумя степенями свободы, ТМФ, 2016, том 187, номер 1, 39-57

DOI: https://doi.org/10.4213/tmf8950

Использование Общероссийского математического портала Math-Net.Ru подразумевает, что вы прочитали и согласны с пользовательским соглашением http://www . mathnet.ru/rus/agreement

Параметры загрузки :

IP: 44.207 .124 .84

26 апреля 2023 г., 12:35:13

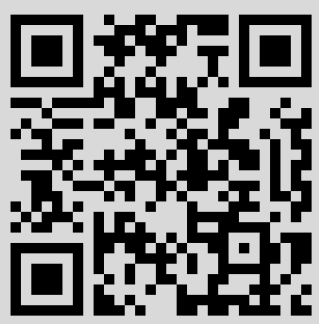




\section{"КВАНТОВАНИЯ" ИЗОМОНОДРОМНОЙ ГАМИЛЬТОНОВОЙ СИСТЕМЫ ГАРНЬЕ С ДВУМЯ СТЕПЕНЯМИ СВОБОДЫ}

Построены решения аналогов временны́х уравнений Шредингера, соответствующих изомонодромной полиномиальной гамильтоновой системе Гарнье с двумя степенями свободы. Они определены решениями линейных обыкновенных дифференциальных уравнений, условием совместности которых является данная система Гарнье. Эти решения с помощью явных замен сводятся также к решениям уравнений Белавина-Полякова-Замолодчикова с четырьмя временами и двумя пространственными переменными.

Ключевые слова: уравнения Шредингера, гамильтоновость, изомонодромные деформации, система Гарнье, уравнения Белавина-Полякова-Замолодчикова, уравнения Пенлеве.

DOI: $10.4213 / \operatorname{tmf} 8950$

\section{1. ВВЕДЕНИЕ}

В теории обыкновенных дифференциальных уравнений (ОДУ) типа Пенлеве в последние годы выделилась тема связей между линейными уравнениями метода изомонодромных деформаций (ИДМ), которые совместны на этих нелинейных ОДУ, и линейными дифференциальными уравнениями в частных производных квантовой механики и квантовой теории поля [1]-[27]. Хронологически первой по этой теме является, по-видимому, статья [1], где установлен следующий факт: шесть канонических ОДУ Пенлеве $\lambda_{t t}^{\prime \prime}=f_{j}\left(t, \lambda, \lambda_{t}^{\prime}\right), j=1, \ldots, 6$, эквивалентны гамильтоновым системам

$$
\lambda_{t}^{\prime}=H_{\mu}^{\prime}(t, \lambda, \mu), \quad \mu_{t}^{\prime}=-H_{\lambda}^{\prime}(t, \lambda, \mu)
$$

с такими гамильтонианами $H=H_{j}(t, \lambda, \mu)$, что решения уравнений ИДМ [28]

$$
W_{x x}^{\prime \prime}=P(t, \lambda(t), \mu(t) ; x) W, \quad W_{t}^{\prime}=A(t, \lambda(t), \mu(t) ; x) W_{x}^{\prime}-\frac{A_{x}^{\prime}(t, \lambda(t), \mu(t) ; x)}{2} W,
$$

Исследование Б. И. Сулейманова выполнено за счет гранта Российского научного фонда (проект № 14-11-00078).

* Омский государственный технический университет, Омск, Россия.

${ }^{\dagger}$ Институт математики с ВЦ УНЦ РАН, Уфа, Россия.

E-mail: bisul@mail.ru 
условием совместности которых являются соответствующие системы (1), с помощью явных замен вида $\Psi=W e^{S(t, x)}$ переводятся в решения уравнений

$$
\varepsilon \frac{\partial \Psi}{\partial t}=H\left(t, x, \varepsilon \frac{\partial}{\partial x}\right) \Psi, \quad \varepsilon=1 .
$$

Правые части уравнений (2), уже не содержащие зависимости от $\lambda(t)$ и $\mu(t)$, при конкретном выборе очередности действий операторов умножения на переменную $x$ и дифференцирования по ней задаются гамильтонианами $H=H_{j}(t, \lambda, \mu), j=1, \ldots, 6$, гамильтоновых систем (1). Выбор другой очередности позволяет [5] символически записать эти шесть линейных эволюционных уравнений как уравнения

$$
\varepsilon \frac{\partial \Psi}{\partial t}=H\left(t, x,-\varepsilon \frac{\partial}{\partial x}\right) \Psi .
$$

Из квантово-механических уравнений Шредингера, зависящих от постоянной Планка $h=2 \pi \hbar=-2 \pi i \varepsilon$, эволюционные уравнения (3) получаются в результате формальной замены $\varepsilon=1$. Далее в настоящей статье подобные аналоги уравнения Шредингера, которые не зависят от постоянной Планка, следуя терминологии, введенной в работе [2], будем называть “квантованиями” соответствующих гамильтоновых систем.

ЗАмечание 1. "Квантования" (2) с $\varepsilon=1$ встречаются в задачах диффузии [29], [30]. Рассмотренная выше связь между “квантованием" (2) ОДУ Пенлеве и соответствующими уравнениями ИДМ использовалась в работах [9], [10] и в серии публикаций Руманова [19]-[22] при исследовании некоторых проблем теоретико-вероятностного характера. Но в ряде частных случаев эта связь оказывается полезной (см. работы [3], [4], [17], [26]) и для построения решений квантово-механических временнь́х уравнений Шредингера (3), в которых $\varepsilon=i \hbar$.

Связь линейных уравнений ИДМ с уравнениями квантовой теории поля была выявлена в [6]. В этой статье показано, что совместные уравнения ИДМ

$$
\Phi_{x}^{\prime}=\sum_{i=1}^{m} \frac{A_{i}}{x-t_{i}} \Phi, \quad \Phi_{t_{i}}^{\prime}=-\frac{A_{i}}{x-t_{i}} \Phi
$$

для решений систем Шлезингера в матрицах $A_{i}$ размера $2 \times 2$ с помощью замены

$$
\Psi=\tau\left(t_{1}, \ldots, t_{m}\right) \Phi, \quad(\ln \tau)_{t_{i}}^{\prime}=\sum_{j \neq i}^{m} \operatorname{tr} \frac{A_{j} A_{i}}{t_{i}-t_{j}},
$$

переводятся в систему

$$
\begin{gathered}
\sum_{i} \frac{\Psi_{t_{i}}^{\prime}}{x-t_{i}}=\Psi_{x x}^{\prime \prime}-\sum_{i} \frac{\Delta_{i} \Psi}{\left(x-t_{i}\right)^{2}}, \quad \sum_{i}\left(x-t_{i}\right) \Psi_{t_{i}}^{\prime}=\left(\sum_{i} \Delta_{i}-A_{\infty}-\Delta_{\infty}\right) \Psi \\
\sum_{i} \Psi_{t_{i}}^{\prime}=-\Psi_{x}^{\prime}
\end{gathered}
$$

где $\Delta_{i}=-\operatorname{det} A_{i}, \Delta_{\infty}-$ постоянные, $A_{\infty}$ - постоянная матрица. Напомним, что системы Шлезингера вида

$$
\frac{\partial A_{j}}{\partial t_{i}}=\frac{\left[A_{i}, A_{j}\right]}{t_{i}-t_{j}}, \quad i \neq j, \quad \frac{\partial A_{i}}{\partial t_{i}}=-\sum_{j \neq i} \frac{\left[A_{i}, A_{j}\right]}{t_{i}-t_{j}}, \quad i, j=\overline{1, m},
$$

были открыты [31] именно в качестве условия совместности ОДУ (4). 
При условии диагональности матрицы $A_{\infty}$ уравнения для $\Psi$ совпадают (см. работу [6]) с пространственно одномерными уравнениями Белавина-Полякова-Замолодчикова (БПЗ) [32], [33] минимальной модели двумерной квантовой теории поля с конформной группой симметрий, для которых центральный заряд алгебры Вирасоро равен единице. В приложении В статьи [6] также указано, что та же $\tau$-функция, что и выше, и совместные решения $\Phi$ линейных ОДУ (4) задают матрицы

$$
M\left(x_{1}, x_{2}\right)=\tau \Phi^{-1}\left(x_{2}\right) \Phi\left(x_{1}\right), \quad x_{1}=x, \quad x_{2}=y,
$$

размера $2 \times 2$, удовлетворяющие четырем линейным дифференциальным уравнениям в частных производных с дифференцированием уже по двум пространственным независимым переменным $x_{1}, x_{2}$. Простой заменой (см. раздел 2 настоящей статьи) эти скалярные уравнения сводятся к уравнениям БПЗ с двумя пространственными переменными.

Формула (6) (см. также статью [34]) позднее послужила основой построения в [5] совместных решений "квантований"

$$
\varepsilon \frac{\partial \Psi}{\partial t_{i}}=H_{t_{i}}\left(t_{1}, t_{2}, x_{1}, x_{2},-\varepsilon \frac{\partial}{\partial x_{1}},-\varepsilon \frac{\partial}{\partial x_{2}}\right) \Psi, \quad i=1,2,
$$

определяемых гамильтонианами $H_{t_{i}}\left(t_{1}, t_{2}, q_{1}, q_{2}, p_{1}, p_{2}\right)$ гамильтоновых систем

$$
\left(q_{j}\right)_{t_{i}}^{\prime}=\left(H_{t_{i}}\right)_{p_{j}}^{\prime}, \quad\left(p_{j}\right)_{t_{i}}^{\prime}=-\left(H_{t_{i}}\right)_{q_{j}}^{\prime}, \quad j=1,2,
$$

которые представляют собой изомонодромные высшие аналоги первого и второго ОДУ Пенлеве. Данные решения эволюционных уравнений (7) выражаются через совместные решения соответствующих линейных уравнений ИДМ формулой типа (6). Естественным выглядит вопрос: можно ли таким образом строить решения уравнений (7) для других изомонодромных гамильтоновых систем с двумя степенями свободы? В настоящей статье показано, что ответ на него положителен для полиномиальной гамильтоновой системы Гарнье, которая была введена в рассмотрение в [35] и которая с помощью явных преобразований [35]-[37] сводится к давно известной изомонодромной системе Гарнье [28].

ЗАмечАниЕ 2. Список известных на сегодня гамильтоновых систем с двумя степенями свободы, к которым применим ИДМ, можно найти в работах [38]-[40]. Хорошо известно, что система Гарнье возглавляет целую иерархию таких систем их можно получить из системы Гарнье с помощью процедуры последовательного вырождения [37], [40]. С применением этой процедуры рассматриваемые далее конструкции, вероятно, можно расширить и на всю указанную иерархию. Отметим, что системы, исследованные в [5], являются двумя низшими членами данной иерархии.

В своих построениях мы опираемся на упомянутые выше результаты работы [6]. Их изложение и уточнение связи этих результатов с уравнениями БПЗ с двумя пространственными переменными производится в начале следующего раздела статьи. Далее в разделе 2 показывается, что в случае четырех времен данные уравнения БПЗ эквивалентны совместной системе эволюционных уравнений, два из которых суть "квантования" вида (7) гамильтоновой изомонодромной системы Гарнье с двумя степенями свободы в форме, выписанной Окамото в [36] (ниже она называется системой Гарнье-Окамото $(\Gamma О))$. Эта система связана с частным случаем системы $(5)$ с четырьмя временами $(m=4)$ [36], [41] . Однако, как подчеркивается 
в заключительном п. 2.5 раздела 2 настоящей статьи, сведение всех решений упомянутой системы ГО с двумя степенями свободы к решениям систем Шлезингера с четырьмя временами $t_{m}$ в явном виде до сих пор не описано.

Представление о сложности и тонкости вопроса о связи между системами Шлезингера и Гарнье, на которую указывалось еще самим Гарнье [42], можно составить на основе обзора [43]. Между тем в разделах 2 и 3 нашей работы решения эволюционных уравнений (7), определяемых общей системой ГО, построены в терминах решений линейных ОДУ (4), коэффициенты которых заданы именно всевозможными решениями общей системы Шлезингера (5) с четырьмя временами $t_{m}$. K последним упомянутым решениям, как показано в разделе 3 , сводятся все решения полиномиальной системы Гарнье с двумя степенями свободы. В этом разделе выводится также формула (46), связывающая системы ГО и полиномиальные системы Гарнье для довольно широкого класса случаев. Однако, как продемонстрировано в конце раздела 3, однозначного соответствия между решениями этих двух систем данная простая формула во всех случаях не дает.

Но с использованием квантового аналога формулы (46) - замены (56) - в заключительном разделе показано, что система уравнений (7), определяемых общей системой ГО, эквивалентна совместной паре (7), задаваемой гамильтонианами общей полиномиальной системы Гарнье. Таким образом, решения этих "квантований" выписываются в терминах совместных решений систем линейных ОДУ, коэффициенты которых задаются как раз всевозможными решениями соответствующей полиномиальной системы Гарнье.

\section{2. СИСТЕМЫ ШЛЕЗИНГЕРА, УРАВНЕНИЯ БПЗ С ДВУМЯ ПРОСТРАНСТВЕННЫМИ ПЕРЕМЕННЫМИ И "КВАНТОВАНИЯ" СИСТЕМЫ ГО}

2.1. Вторую группу уравнений в системе Шлезингера (5) заменяет условие постоянства по $t_{1}, \ldots, t_{m}$ матрицы $A_{1}+\cdots+A_{m}=A_{\infty}$. Без фактического ограничения общности системы Шлезингера размера $2 \times 2$ рассматривались в статье [6] в предположении, что их решения $B_{i}$ таковы, что $\operatorname{tr} B_{i}=0, \operatorname{det} B_{i}=-\Delta_{i}=-\theta_{i}^{2} / 4$ (постоянные $\pm \theta_{i} / 2$ суть собственные значения матриц $\left.B_{i}\right), \operatorname{det} B_{\infty}=-\Delta_{\infty}$, а матрица $B_{\infty}$ определяется одним из выражений

$$
\begin{aligned}
& B_{\infty}=\frac{1}{2}\left(\begin{array}{cc}
k_{\infty} & 0 \\
0 & -k_{\infty}
\end{array}\right), \\
& B_{\infty}=\left(\begin{array}{ll}
0 & 0 \\
1 & 0
\end{array}\right) .
\end{aligned}
$$

2.2. В статье [6] (см. приложение В) выписана система четырех уравнений

$$
\begin{gathered}
\sum_{i}\left(\frac{1}{x-t_{i}}+\frac{1}{y-x}\right) M_{t_{i}}^{\prime}=M_{x x}^{\prime \prime}-\sum_{i} \frac{\Delta_{i}}{\left(x-t_{i}\right)^{2}} M \\
\sum_{i}\left(\frac{1}{y-t_{i}}+\frac{1}{x-y}\right) M_{t_{i}}^{\prime}=M_{y y}^{\prime \prime}-\sum_{i} \frac{\Delta_{i}}{\left(y-t_{i}\right)^{2}} M \\
\sum_{i} M_{t_{i}}^{\prime}+M_{x}^{\prime}+M_{y}^{\prime}=0, \quad \sum_{i} t_{i} M_{t_{i}}^{\prime}+x M_{x}^{\prime}+y M_{y}^{\prime}=\left(\Delta_{\infty}-\sum_{i} \Delta_{i}\right) M
\end{gathered}
$$


решением которой является матрица (6). Результат $Y(t, x, y)$ замены

$$
M=(x-y) \prod_{i=1}^{m}\left(\left(x-t_{i}\right)\left(y-t_{i}\right)\right)^{\theta_{i} / 2} e^{S(t)} Y, \quad S_{t_{i}}=\frac{\theta_{i}}{2} \sum_{j \neq i} \frac{\theta_{j}}{t_{i}-t_{j}},
$$

удовлетворяет системе БПЗ с двумя пространственными переменными (см. статью [44]):

$$
\begin{gathered}
\sum_{i=1}^{m} \frac{Y_{t_{i}}^{\prime}}{x-t_{i}}=Y_{x x}^{\prime \prime}+\frac{Y_{x}-Y_{y}}{x-y}+\sum_{i=1}^{m} \frac{\theta_{i}}{x-t_{i}} Y_{x}^{\prime}, \\
\sum_{i=1}^{m} \frac{Y_{t_{i}}^{\prime}}{y-t_{i}}=Y_{y y}^{\prime \prime}+\frac{Y_{x}^{\prime}-Y_{y}^{\prime}}{x-y}+\sum_{i=1}^{m} \frac{\theta_{i}}{y-t_{i}} Y_{y}^{\prime}, \\
\sum_{i=1}^{m} Y_{t_{i}}^{\prime}+Y_{x}^{\prime}+Y_{y}^{\prime}=0, \quad \sum_{i=1}^{m} t_{i} Y_{t_{i}}^{\prime}+x Y_{x}^{\prime}+y Y_{y}^{\prime}=\lambda Y=\left(\Delta_{\infty}-\left(1+\sum_{i=1}^{m} \frac{\theta_{i}}{2}\right)^{2}\right) Y .
\end{gathered}
$$

2.3. Решения $B_{i}$ системы Шлезингера (5) из п. 2.1 сдвигами

$$
Q_{i}=\left(\begin{array}{ll}
q_{11}^{i}(t) & q_{12}^{i}(t) \\
q_{21}^{i}(t) & q_{22}^{i}(t)
\end{array}\right)=B_{i}+\frac{\theta_{i}}{2}\left(\begin{array}{ll}
1 & 0 \\
0 & 1
\end{array}\right)
$$

преобразуются в решения $Q_{i}$ этой же системы, удовлетворяющие следующим условиям.

УсловиЕ 1. Собственные значения матриц $Q_{i}(t)$ суть нуль и постоянная $\theta_{i}$.

При этом преобразование $Z=\Phi \prod_{i=1}^{4}\left(x-t_{i}\right)^{\theta_{i} / 2}$ переводит решения $\Phi$ системы (4) с матрицами $A_{i}=B_{i}$ в решения $Z$ этой же системы, но уже с матрицами $A_{i}=Q_{i}$.

Далее рассматриваются эти решения $Q_{i}$ уравнений Шлезингера (5) в случае четырех времен $t_{i}$ с последующей фиксацией переменных $t_{3}$ и $t_{4}$ :

$$
t_{3}=1, \quad t_{4}=0 .
$$

ЗАмЕчАНИЕ 3 . То, что мы фиксируем $t_{3}$ и $t_{4}$, по сути не ограничивает общности рассмотрения. Действительно, совместные решения $A_{k}, k=1, \ldots, 4$, уравнений (5) с независимыми переменными $t_{1}, t_{2}$ при ограничении (13) можно рассматривать как начальные данные (при $t_{3}=1$ ) для уравнений $(5)$ с независимой переменной $t_{3}$. Решение начальной задачи для уравнений Шлезингера (5) с независимой переменной $t_{3}$ и этими начальными данными можно, в свою очередь, рассматривать как начальные данные при $t_{4}=0$ для системы уравнений (5) с независимой переменной $t_{4}$, а решение этой начальной задачи является совместным решением всех уравнений (5).

УсловиЕ 2. В предположении, что решения $B_{i}$ системы Шлезингера (5) из раздела 2 определяют постоянную матрицу $B_{\infty}$ вида (8), справедливо соотношение

$$
-\sum_{i=1}^{4} Q_{i}(t)=\left(\begin{array}{cc}
\chi & 0 \\
0 & \chi+\theta_{\infty}-1
\end{array}\right)
$$

где $\theta_{\infty}=k_{\infty}+1$ не зависит от $t_{i}$ и $\chi=-\left(\sum_{i=1}^{4} \theta_{i}+\theta_{\infty}-1\right) / 2$. 
Пусть также решения $Q_{i}(t)$ системы Шлезингера удовлетворяют следующим условиям.

УсловиЕ 3. Элемент $q_{12}(x, t)$ матрицы

$$
Q(x, t)=\left(\begin{array}{ll}
q_{11}(x, t) & q_{12}(x, t) \\
q_{21}(x, t) & q_{22}(x, t)
\end{array}\right)=\sum_{i=1}^{4} \frac{Q_{i}}{x-t_{i}}
$$

очевидно, равный

$$
q_{12}(x, t)=\frac{X(t) x^{2}+a_{1}(t) x+a_{2}(t)}{T(x, t)}=\frac{\sum_{i=1}^{4} t_{i} q_{12}^{i} x^{2}+a_{1}(t) x+a_{2}(t)}{\prod_{i=1}^{4}\left(x-t_{i}\right)},
$$

таков, что функция $X(t)=\sum_{i=1}^{4} t_{i} q_{12}^{i}$ не равна тождественно нулю.

УсловиЕ 4 . Все нули $x(t)=\lambda_{j}(t)$ элемента $q_{12}(x, t)$ простые.

Из условий 2-4 следует (см. [36] и книгу [41]), что: а) $q_{12}(x, t)$ имеет два простых нуля и задается формулой

$$
q_{12}(x, t)=\sum_{i=1}^{4} \frac{q_{12}^{i}(t)}{x-t_{i}}=X(t) \frac{\Lambda(x, t)}{T(x, t)}=X(t) \frac{\left(x-\lambda_{1}(t)\right)\left(x-\lambda_{2}(t)\right)}{T(x, t)} ;
$$

б) компонента $z(x, t)=z_{1}(x, t)$ любого вектора $Z=\left(z_{1}, z_{2}\right)$, являющегося решением первой из систем ОДУ $(4)$ с матрицами $A_{i}(t)=Q_{i}(t)$, удовлетворяет линейному ОДУ

$$
\begin{aligned}
z_{x x}^{\prime \prime}= & \left(\sum_{i=1}^{4} \frac{\theta_{i}-1}{x-t_{i}}+\sum_{k=1}^{2} \frac{1}{x-\lambda_{k}}\right) z_{x}^{\prime}- \\
& -\left(\frac{\kappa}{x(x-1)}-\sum_{i=1}^{2} \frac{t_{i}\left(t_{i}-1\right) K_{i}}{x(x-1)\left(x-t_{i}\right)}+\sum_{k=1}^{2} \frac{\lambda_{k}\left(\lambda_{k}-1\right) \mu_{k}}{x(x-1)\left(x-\lambda_{k}\right)}\right) z,
\end{aligned}
$$

где

$$
\kappa=\frac{1}{4}\left[\left(\sum_{i=1}^{4} \theta_{i}-1\right)^{2}-\theta_{\infty}^{2}\right], \quad \mu_{k}=\sum_{i=1}^{4} \frac{q_{11}^{i}}{\lambda_{k}-t_{i}}
$$

Совместность систем уравнений (4) означает, что наряду с уравнением (16) компонента $z(x, t)$ удовлетворяет также эволюционным уравнениям первого порядка

$$
z_{t_{i}}^{\prime}=C_{i}(x, t) z_{x}^{\prime}+J_{i}(x, t) z=s_{i}(t) \frac{T(x, t)}{\left(x-t_{i}\right) \Lambda(x, t)} z_{x}^{\prime}+J_{i}(x, t) z, \quad i=1,2 .
$$

В свою очередь, замена

$$
z(x, t)=\prod_{i=1}^{4}\left(x-t_{i}\right)^{\left(\theta_{i}-1\right) / 2}\left(x-\lambda_{1}(t)\right)^{1 / 2}\left(x-\lambda_{2}(t)\right)^{1 / 2} v(x, t)
$$


преобразует решение $z(x, t)$ уравнений $(16)$ и $(18)$ в решение системы

$$
\begin{aligned}
& v_{x x}^{\prime \prime}= {\left[\frac{c_{3}}{x^{2}}+\frac{c_{4}}{(x-1)^{2}}+\frac{c_{5}}{x(x-1)}+\sum_{i=1}^{2}\left(\frac{c_{i}}{\left(x-t_{i}\right)^{2}}+\frac{\alpha_{i}(t)}{x(x-1)\left(x-t_{i}\right)}\right)+\right.} \\
&\left.+\sum_{j=1}^{2}\left(\frac{3}{4\left(x-\lambda_{j}(t)\right)^{2}}+\frac{\beta_{j}(t)}{x(x-1)\left(x-\lambda_{j}(t)\right)}\right)\right] v, \quad\left(c_{j}\right)_{t_{i}}^{\prime}=0, \\
& v_{t_{i}}^{\prime}=C_{i}(x, t) v_{x}^{\prime}+\left[-\frac{\left(C_{i}(x, t)\right)_{x}^{\prime}}{2}+\gamma_{i}(t)\right] v .
\end{aligned}
$$

Отсюда следует (см. [28], [36]), что условие совместности ОДУ (16) с уравнением первого порядка (18) определяется совместными между собой гамильтоновыми системами с двумя степенями свободы:

$$
\frac{\partial \lambda_{k}}{\partial t_{j}}=\frac{\partial K_{j}}{\partial \mu_{k}}, \quad \frac{\partial \mu_{k}}{\partial t_{j}}=-\frac{\partial K_{j}}{\partial \lambda_{k}}, \quad j, k=1,2,
$$

где $\lambda_{i}$ суть рассмотренные выше нули элемента $q_{12}(x, t)$, импульсы $\mu_{i}$ задаются формулами (17), а гамильтонианы $K_{i}$ - формулами

$$
\begin{aligned}
K_{i} & =K_{i}\left(t_{1}, t_{2}, \lambda_{1}, \lambda_{2}, \mu_{1}, \mu_{2} ; \theta_{1}, \theta_{2}, \theta_{3}, \theta_{4}, \kappa\right)= \\
& =M_{i} \sum_{k=1}^{2} M^{k, i}\left[\mu_{k}^{2}-\left(\sum_{m=1}^{2} \frac{\theta_{m}-\delta_{i m}}{\lambda_{k}-t_{m}}+\frac{\theta_{3}}{\lambda_{k}-1}+\frac{\theta_{4}}{\lambda_{k}}\right) \mu_{k}+\frac{\kappa}{\lambda_{k}\left(\lambda_{k}-1\right)}\right],
\end{aligned}
$$

в которых $\delta_{m i}-$ символ Кронекера,

$$
M_{i}=-\frac{\left(\lambda_{1}-t_{i}\right)\left(\lambda_{2}-t_{i}\right)}{\left(t_{i}-t_{i+1}\right)\left(t_{i}-1\right) t_{i}}, \quad M^{k, i}=\frac{\left(\lambda_{k}-t_{i+1}\right)\left(\lambda_{k}-1\right) \lambda_{k}}{\lambda_{k}-\lambda_{k+1}} .
$$

Уравнения (19) представляют собой изомонодромную гамильтонову систему ГО с двумя степенями свободы [41], [45].

ЗАмЕчание 4. На самом деле в работах [36], [41] условие 3 явно не сформулировано, но налагаются дополнительные ограничения на соответствующие матрицы $Q_{i}$. В частности, в статье [41] (см. раздел 6.2) приведено следующее ограничение.

Условие 5. Собственные значения матриц $Q_{i}$ не являются целыми числами.

2.4. При $m=4$ и выполнении равенства (13) система (10) эквивалентна системе

$$
\begin{aligned}
t_{1}\left(t_{1}\right. & -1)\left(t_{1}-t_{2}\right) Y_{t_{1}}^{\prime}=\frac{\left(x-t_{1}\right)\left(y-t_{1}\right)\left(x-t_{2}\right)(x-1) x}{y-x} \times \\
& \times\left[Y_{x x}^{\prime \prime}+Y_{x}^{\prime}\left(\frac{\theta_{1}}{x-t_{1}}+\frac{\theta_{2}+1}{x-t_{2}}+\frac{\theta_{3}+1}{x-1}+\frac{\theta_{4}+1}{x}\right)-\frac{\lambda}{x(x-1)} Y\right]- \\
& -\frac{\left(x-t_{1}\right)\left(y-t_{1}\right)\left(y-t_{2}\right)(y-1) y}{y-x} \times \\
& \times\left[Y_{y y}^{\prime \prime}+Y_{y}^{\prime}\left(\frac{\theta_{1}}{y-t_{1}}+\frac{\theta_{2}+1}{y-t_{2}}+\frac{\theta_{3}+1}{y-1}+\frac{\theta_{4}+1}{y}\right)-\frac{\lambda}{y(y-1)} Y\right],
\end{aligned}
$$




$$
\begin{aligned}
t_{2}\left(t_{2}\right. & -1)\left(t_{2}-t_{1}\right) Y_{t_{2}}^{\prime}=\frac{\left(x-t_{2}\right)\left(y-t_{2}\right)\left(x-t_{1}\right)(x-1) x}{y-x} \times \\
& \times\left[Y_{x x}^{\prime \prime}+Y_{x}^{\prime}\left(\frac{\theta_{1}+1}{x-t_{1}}+\frac{\theta_{2}}{x-t_{2}}+\frac{\theta_{3}+1}{x-1}+\frac{\theta_{4}+1}{x}\right)-\frac{\lambda}{x(x-1)} Y\right]- \\
& -\frac{\left(x-t_{2}\right)\left(y-t_{2}\right)\left(y-t_{1}\right)(y-1) y}{y-x} \times \\
& \times\left[Y_{y y}^{\prime \prime}+Y_{y}^{\prime}\left(\frac{\theta_{1}+1}{y-t_{1}}+\frac{\theta_{2}}{y-t_{2}}+\frac{\theta_{3}+1}{y-1}+\frac{\theta_{4}+1}{y}\right)-\frac{\lambda}{y(y-1)} Y\right],
\end{aligned}
$$

дополненной уравнениями (11). Уравнения (21), (22) суть "квантования" гамильтоновой системы ГО (19) двух переменных, поскольку в силу операторных соотношений

$$
\frac{\partial}{\partial x} x-x \frac{\partial}{\partial x}=1, \quad \frac{\partial}{\partial y} y-y \frac{\partial}{\partial y}=1
$$

при подходящем выборе порядка действия операторов дифференцирования по переменным $x, y$ и умножения на многочлены от этих переменных данные эволюционные уравнения символически можно представить в следующем виде $(\varepsilon=1)$ :

$$
\varepsilon \frac{\partial Y}{\partial t_{i}}=K_{i}\left(t_{1}, t_{2}, x, y,-\varepsilon \frac{\partial}{\partial x},-\varepsilon \frac{\partial}{\partial y} ; \theta_{1}, \theta_{2}, \theta_{3}, \theta_{4}, \kappa\right) Y, \quad i=1,2,
$$

где $K_{i}\left(t_{1}, t_{2}, \lambda_{1}, \lambda_{2}, \mu_{1}, \mu_{2} ; \theta_{1}, \theta_{2}, \theta_{3}, \theta_{4}, \kappa\right)$ суть гамильтонианы (20) этой гамильтоновой системы.

ЗАмЕчАниЕ 5. При подходящем выборе порядка действия указанных выше операторов уравнения (21), (22) можно также представить как “квантования" вида (23) (при $\varepsilon=1$ )

$$
\varepsilon \frac{\partial Y}{\partial t_{i}}=K_{i}\left(t_{1}, t_{2}, x, y,-\varepsilon \frac{\partial}{\partial x},-\varepsilon \frac{\partial}{\partial y} ; \hat{\theta}_{1}, \hat{\theta}_{2}, \hat{\theta}_{3}, \hat{\theta}_{4}, \hat{\kappa}\right) Y, \quad i=1,2,
$$

гамильтоновых систем ГО (19), которые вместо постоянных $\theta_{k}, k=1, \ldots, 4$, и $\kappa$ зависят от любых других постоянных $\hat{\theta}_{k}, k=1, \ldots, 4$, и $\hat{\kappa}$. В частности, для всех гамильтонианов (20) уравнения $(21),(22)$ можно символически записать в виде (при $\varepsilon=1)$

$$
\varepsilon Y_{t_{i}}=K_{i}\left(t_{1}, t_{2}, x, y, \varepsilon \frac{\partial}{\partial x}, \varepsilon \frac{\partial}{\partial y} ; \theta_{1}, \theta_{2}, \theta_{3}, \theta_{4}, \kappa\right) Y, \quad i=1,2 .
$$

2.5. Выше рассмотрена конструкция, которая позволяет выписать в явном виде совместные решения эволюционных уравнений (21), (22) в терминах совместных решений линейных систем ОДУ (4) при $m=4$. Коэффициенты последних задаются множеством решений нелинейных систем Шлезингера (5). Таким образом, имеется однозначное соответствие между построенными совместными решениями эволюционных уравнений $(21),(22)$ и данным множеством. Поэтому возникает естественное желание выразить множество решений системы Шлезингера через решения гамильтоновой системы ГО (19) с теми же независимыми переменными $t_{1}, t_{2}$. Однако вопрос о связи между системами Шлезингера и Гарнье не так прост. Не совсем точны, например, высказываемые иногда утверждения о том, что в статье [36] описана эквивалентность этих систем. Даже в случае двух степеней свободы системы ГО в [36] 
эквивалентность доказана лишь при выполнении для решений $B_{i}$ системы Шлезингера равенства (8) с ненулевыми постоянными $k_{\infty}$ и ряда других предположений. В частности, в этой статье не показано, что все возможные решения системы ГО с двумя степенями свободы сводятся к решениям систем Шлезингера размера $2 \times 2$. До сих пор в явном виде это нигде не было не сделано.

В следующем разделе показано, каким образом общие решения полиномиальной гамильтоновой системы Гарнье с двумя степенями свободы можно свести к решениям систем Шлезингера размера $2 \times 2$ безо всяких дополнительных предположений. Во многом этот раздел основан на результатах недавних работ [38], [40], но в пп. 3.3-3.5 излагаются также весьма существенные дополнения к этим результатам.

\section{3. ПОЛИНОМИАЛЬНАЯ СИСТЕМА ГАРНЬЕ}

3.1. Полиномиальную систему Гарнье составляют совместные гамильтоновы системы

$$
\left(q_{j}\right)_{t_{i}}^{\prime}=\left(H_{\mathrm{Gar}, t_{i}}\right)_{p_{j}}^{\prime}, \quad\left(p_{j}\right)_{t_{i}}^{\prime}=-\left(H_{\mathrm{Gar}, t_{i}}\right)_{q_{j}}^{\prime}, \quad i, j=1,2,
$$

с гамильтонианами $H_{\mathrm{Gar}, t_{i}}$ и $H_{\mathrm{Gar}, t_{i+1}}$, первый из которых задается формулой

$$
\begin{aligned}
t_{i}\left(t_{i}-1\right) H_{\mathrm{Gar}, t_{i}}= & q_{i}\left(q_{i}-1\right)\left(q_{i}-t_{i}\right) p_{i}^{2}+\left[\left(\theta^{0}+\theta^{t_{i+1}}+1\right) q_{i}\left(q_{i}-1\right)-\right. \\
& \left.-\left(2 \theta_{2}^{\infty}+\theta^{1}+\theta^{0}+\theta^{t_{i}}+\theta^{t_{i+1}}+1\right) q_{i}\left(q_{i}-t_{i}\right)+\theta^{t_{i}}\left(q_{i}-1\right)\left(q_{i}-t_{i}\right)\right] p_{i}+ \\
& +\theta_{2}^{\infty}\left(\theta_{2}^{\infty}+\theta^{1}\right) q_{i}+\left(2 q_{i} p_{i}+q_{i+1} p_{i+1}-\theta^{1}-2 \theta_{2}^{\infty}\right) q_{i} q_{i+1} p_{i+1}- \\
& -\frac{1}{t_{i}-t_{i+1}}\left[t_{i}\left(t_{i}-1\right)\left(p_{i} q_{i}+\theta^{t_{i}}\right) p_{i} q_{i+1}-\right. \\
& -t_{i}\left(t_{i+1}-1\right)\left(2 p_{i} q_{i}+\theta^{t_{i}}\right) p_{i+1} q_{i+1}+ \\
& \left.+t_{i+1}\left(t_{i}-1\right) q_{i}\left(p_{i+1}^{2} q_{i+1}+\theta^{t_{i+1}}\left(p_{i+1}-p_{i}\right)\right)\right]
\end{aligned}
$$

а второй получается из (25) заменами $t_{1} \leftrightarrow t_{2}, q_{1} \leftrightarrow q_{2}, p_{1} \leftrightarrow p_{2}$. В этих гамильтонианах $\theta^{0}, \theta^{1}, \theta^{t_{1}}, \theta^{t_{2}}, \theta_{1}^{\infty}, \theta_{2}^{\infty}$ суть постоянные, которые связаны так называемым соотношением Фукса:

$$
\theta^{0}+\theta^{1}+\theta^{t_{1}}+\theta^{t_{2}}+\theta_{1}^{\infty}+\theta_{2}^{\infty}=0,
$$

соответствующим выписываемой ниже системе ИДМ (36).

Первая из гамильтоновых систем (24) представляет собой систему ОДУ

$$
\begin{aligned}
t_{i}\left(t_{i}-1\right)\left(q_{i}\right)_{t_{i}}^{\prime}= & 2 p_{i} q_{i}\left[\left(q_{i}-1\right)\left(q_{i}-t_{i}\right)-\frac{t_{i}\left(t_{i}-1\right)}{t_{i}-t_{i+1}} q_{i+1}\right]+ \\
& +2 p_{i+1} q_{i} q_{i+1}\left[q_{i}+\frac{t_{i}\left(t_{i+1}-1\right)}{t_{i}-t_{i+1}}\right]-\left(\theta^{1}+2 \theta_{2}^{\infty}\right) q_{i}^{2}- \\
& -\left(1+\theta^{0}+\theta^{t_{i}}+\theta^{t_{i+1}}\right) q_{i}+\left(1+\theta^{1}+2 \theta_{2}^{\infty}+\theta^{0}+\theta^{t_{i+1}}\right) t_{i} q_{i}+ \\
& +t_{i} \theta^{t_{i}}+\frac{t_{i}-1}{t_{i}-t_{i+1}}\left[t_{i+1} \theta^{t_{i+1}} q_{i}-t_{i} \theta^{t_{i}} q_{i+1}\right], \\
t_{i}\left(t_{i}-1\right)\left(q_{i+1}\right)_{t_{i}}^{\prime}= & 2 p_{i} q_{i} q_{i+1}\left[q_{i}+\frac{t_{i}\left(t_{i+1}-1\right)}{t_{i}-t_{i+1}}\right]+2 p_{i+1} q_{i} q_{i+1}\left[q_{i+1}-\frac{t_{i+1}\left(t_{i}-1\right)}{t_{i}-t_{i+1}}\right]- \\
& -\left(\theta^{1}+2 \theta_{2}^{\infty}\right) q_{i} q_{i+1}-\frac{t_{i+1}\left(t_{i}-1\right) \theta^{t_{i+1}} q_{i}}{t_{i}-t_{i+1}}+\frac{t_{i}\left(t_{i+1}-1\right) \theta^{t_{i}} q_{i+1}}{t_{i}-t_{i+1}}, \quad(28)
\end{aligned}
$$




$$
\begin{aligned}
& t_{i}\left(t_{i}-1\right)\left(p_{i}\right)_{t_{i}}^{\prime}=-p_{i}^{2}\left[3 q_{i}^{2}-2\left(t_{i}+1\right) q_{i}+t_{i}-\frac{t_{i}\left(t_{i}-1\right)}{t_{i}-t_{i+1}} q_{i+1}\right]- \\
& -2 p_{i+1} p_{i} q_{i+1}\left[2 q_{i}+\frac{t_{i}\left(t_{i+1}-1\right)}{t_{i}-t_{i+1}}\right]-p_{i+1}^{2} q_{i+1}\left[q_{i+1}-\frac{t_{i+1}\left(t_{i}-1\right)}{t_{i}-t_{i+1}}\right]+ \\
& +p_{i}\left[2\left(\theta^{1}+2 \theta_{2}^{\infty}\right) q_{i}+\left(1+\theta^{0}+\theta^{t_{i}}+\theta^{t_{i+1}}\right)-\right. \\
& \left.-\left(1+\theta^{1}+2 \theta_{2}^{\infty}+\theta^{0}+\theta^{t_{i+1}}\right) t_{i}-\frac{t_{i+1}\left(t_{i}-1\right) \theta^{t_{i+1}}}{t_{i}-t_{i+1}}\right]+ \\
& +p_{i+1}\left[\left(\theta^{1}+2 \theta_{2}^{\infty}\right) q_{i+1}+\frac{t_{i+1}\left(t_{i}-1\right) \theta^{t_{i+1}}}{t_{i}-t_{i+1}}\right]-\theta_{2}^{\infty}\left(\theta_{2}^{\infty}+\theta^{1}\right), \\
& t_{i}\left(t_{i}-1\right)\left(p_{i+1}\right)_{t_{i}}^{\prime}=p_{i}^{2} q_{i} \frac{t_{i}\left(t_{i}-1\right)}{t_{i}-t_{i+1}}-2 p_{i+1} p_{i} q_{i}\left[q_{i}+\frac{t_{i}\left(t_{i+1}-1\right)}{t_{i}-t_{i+1}}\right]- \\
& -p_{i+1}^{2} q_{i}\left[2 q_{i+1}-\frac{t_{i+1}\left(t_{i}-1\right)}{t_{i}-t_{i+1}}\right]+p_{i} \frac{\theta^{t_{i}} t_{i}\left(t_{i}-1\right)}{t_{i}-t_{i+1}}+ \\
& +p_{i+1}\left[\left(\theta^{1}+2 \theta_{2}^{\infty}\right) q_{i}-\frac{t_{i}\left(t_{i+1}-1\right) \theta^{t_{i}}}{t_{i}-t_{i+1}}\right] \text {. }
\end{aligned}
$$

Вторая из гамильтоновых систем (24) является системой ОДУ

$$
\begin{aligned}
t_{i+1}\left(t_{i+1}-1\right)\left(q_{i}\right)_{t_{i+1}}^{\prime}= & 2 p_{i} q_{i} q_{i+1}\left[q_{i}+\frac{t_{i}\left(t_{i+1}-1\right)}{t_{i}-t_{i+1}}\right]+ \\
& +2 p_{i+1} q_{i} q_{i+1}\left[q_{i+1}-\frac{t_{i+1}\left(t_{i}-1\right)}{t_{i}-t_{i+1}}\right]-\left(\theta^{1}+2 \theta_{2}^{\infty}\right) q_{i} q_{i+1}- \\
& -\frac{1}{t_{i}-t_{i+1}}\left[t_{i+1}\left(t_{i}-1\right) \theta^{t_{i+1}} q_{i}-t_{i}\left(t_{i+1}-1\right) \theta^{t_{i}} q_{i+1}\right], \quad(31) \\
t_{i+1}\left(t_{i+1}-1\right)\left(q_{i+1}\right)_{t_{i+1}}^{\prime}= & 2 p_{i} q_{i} q_{i+1}\left[q_{i+1}-\frac{t_{i+1}\left(t_{i}-1\right)}{t_{i}-t_{i+1}}\right]+ \\
& +2 p_{i+1} q_{i+1}\left[\left(q_{i+1}-1\right)\left(q_{i+1}-t_{i+1}\right)+\frac{t_{i+1}\left(t_{i+1}-1\right)}{t_{i}-t_{i+1}} q_{i}\right]- \\
& -\left(\theta^{1}+2 \theta_{2}^{\infty}\right) q_{i+1}^{2}-\left(1+\theta^{0}+\theta^{t_{i}}+\theta^{t_{i+1}}\right) q_{i+1}+ \\
& +\left(1+\theta^{1}+2 \theta_{2}^{\infty}+\theta^{0}+\theta^{t_{i}}\right) t_{i+1} q_{i+1}+ \\
& +t_{i+1} \theta^{t_{i+1}}+\frac{t_{i+1}-1}{t_{i}-t_{i+1}\left(t_{i+1} \theta^{t_{i+1}} q_{i}-t_{i} \theta^{t_{i}} q_{i+1}\right),} \\
t_{i+1}\left(t_{i+1}-1\right)\left(p_{i}\right)_{t_{i+1}}^{\prime}= & -p_{i}^{2} q_{i+1}\left[2 q_{i}+\frac{t_{i}\left(t_{i+1}-1\right)}{t_{i}-t_{i+1}}\right]- \\
& -2 p_{i+1} p_{i} q_{i+1}\left[q_{i+1}-\frac{t_{i+1}\left(t_{i}-1\right)}{t_{i}-t_{i+1}}\right]-p_{i+1}^{2} q_{i+1} \frac{t_{i+1}\left(t_{i+1}-1\right)}{t_{i}-t_{i+1}}+ \\
+ & -p_{i}\left[\left(\theta^{1}+2 \theta_{2}^{\infty}\right) q_{i+1}+\frac{t_{i+1}\left(t_{i}-1\right) \theta^{t_{i+1}}}{t_{i}-t_{i+1}}\right]- \\
& -p_{i+1} \frac{\theta^{t_{i+1}} t_{i+1}\left(t_{i+1}-1\right)}{t_{i+1}},
\end{aligned}
$$




$$
\begin{aligned}
t_{i+1}\left(t_{i+1}-1\right)\left(p_{i+1}\right)_{t_{i+1}}^{\prime}= & -p_{i}^{2} q_{i}\left[q_{i}+\frac{t_{i}\left(t_{i+1}-1\right)}{t_{i}-t_{i+1}}\right]-2 p_{i+1} p_{i} q_{i}\left[2 q_{i+1}-\frac{t_{i+1}\left(t_{i}-1\right)}{t_{i}-t_{i+1}}\right]- \\
& -p_{i+1}^{2}\left[3 q_{i+1}^{2}-2 q_{i+1}\left(t_{i+1}+1\right)+t_{i+1}+\frac{t_{i+1}\left(t_{i+1}-1\right)}{t_{i}-t_{i+1}} q_{i}\right]+ \\
& +p_{i}\left[\left(\theta^{1}+2 \theta_{2}^{\infty}\right) q_{i}-\frac{t_{i}\left(t_{i+1}-1\right) \theta^{t_{i}}}{t_{i}-t_{i+1}}\right]-\theta_{2}^{\infty}\left(\theta_{2}^{\infty}+\theta^{1}\right)+ \\
& +p_{i+1}\left[2\left(\theta^{1}+2 \theta_{2}^{\infty}\right) q_{i+1}+\left(1+\theta^{0}+\theta^{t_{i}}+\theta^{t_{i+1}}\right)-\right. \\
& \left.-\left(1+\theta^{1}+2 \theta_{2}^{\infty}+\theta^{0}+\theta^{t_{i}}\right) t_{i+1}+\frac{t_{i}\left(t_{i+1}-1\right) \theta^{t_{i}}}{t_{i}-t_{i+1}}\right]
\end{aligned}
$$

3.2. При дополнительном предположении

$$
\theta_{1}^{\infty} \neq \theta_{2}^{\infty}
$$

совместность гамильтоновых систем (24) согласно [40] является условием совместности систем линейных ОДУ Шлезингера

$$
\frac{\partial Z}{\partial x}=\left[\frac{S_{0}}{x}+\frac{S_{1}}{x-1}+\frac{S_{t_{1}}}{x-t_{1}}+\frac{S_{t_{2}}}{x-t_{2}}\right] Z, \quad \frac{\partial Z}{\partial t_{1}}=-\frac{S_{t_{1}}}{x-t_{1}} Z, \quad \frac{\partial Z}{\partial t_{2}}=-\frac{S_{t_{2}}}{x-t_{2}} Z
$$

Здесь матрицы $S_{0}, S_{1}$, и $S_{t_{i}}$ задаются формулами

$$
\begin{gathered}
S_{\xi}=\left(\begin{array}{ll}
1 & 0 \\
0 & u
\end{array}\right)^{-1} P^{-1} \hat{A}_{\xi} P\left(\begin{array}{cc}
1 & 0 \\
0 & u
\end{array}\right), \quad \xi=0,1, t_{1}, t_{2}, \\
\hat{A}_{0}=\left(\begin{array}{ccc}
\theta^{0} & -1+\frac{q_{1}}{t_{1}}+\frac{q_{2}}{t_{2}} \\
0 & 0
\end{array}\right), \\
\hat{A}_{1}=\left(\begin{array}{cc}
\theta^{1}+\theta_{2}^{\infty}-p_{1} q_{1}-p_{2} q_{2} & 1 \\
\left(p_{1} q_{1}+p_{2} q_{2}-\theta_{2}^{\infty}\right) \times & p_{1} q_{1}+p_{2} q_{2}-\theta_{2}^{\infty} \\
\times\left(\theta^{1}+\theta_{2}^{\infty}-p_{1} q_{1}-p_{2} q_{2}\right) & q_{i}
\end{array}\right), \\
\hat{A}_{t_{i}}=\left(\begin{array}{cc}
\theta^{t_{i}+p_{i} q_{i}} & -\frac{q_{i}}{t_{i}} \\
t_{i} p_{i}\left(\theta^{t_{i}}+p_{i} q_{i}\right) & -p_{i} q_{i}
\end{array}\right), \\
P=\left(\begin{array}{cc}
1 & 0 \\
\frac{a}{\theta_{1}^{\infty}-\theta_{2}^{\infty}} & 1
\end{array}\right) .
\end{gathered}
$$

Функция

$$
a=\left(p_{1} q_{1}+p_{2} q_{2}-\theta_{2}^{\infty}\right)\left(p_{1} q_{1}+p_{2} q_{2}-\theta^{1}-\theta_{2}^{\infty}\right)-t_{1} p_{1}\left(\theta^{t_{1}}+p_{1} q_{1}\right)-t_{2} p_{2}\left(\theta^{t_{2}}+p_{2} q_{2}\right)
$$

есть элемент “21” матрицы

$$
\hat{A}_{\infty}=-\hat{A}_{0}-\hat{A}_{1}-\hat{A}_{t_{1}}-\hat{A}_{t_{2}}=\left(\begin{array}{cc}
-\theta^{0}-\theta^{1}-\theta^{t_{1}}-\theta^{t_{2}}-\theta_{2}^{\infty} & 0 \\
a & \theta_{2}^{\infty}
\end{array}\right)
$$


а функция $u=u\left(t_{1}, t_{2}\right)$ - совместное решение двух дифференциальных уравнений

$$
t_{i}\left(t_{i}-1\right) \frac{1}{u} \frac{\partial u}{\partial t_{i}}=q_{i}\left\{2 p_{i}\left(t_{i}-q_{i}\right)+\theta^{1}+2 \theta_{2}^{\infty}\right\}-2 q_{i} p_{i+1} q_{i+1}+t_{i} \theta^{t_{i}}, \quad i=1,2 .
$$

Нетрудно заметить, что собственные значения каждой из матриц $\hat{A}_{\xi}$ равны 0 и $\theta^{\xi}$, а их сумма с учетом соотношения Фукса (26) задается формулой

$$
\hat{A}_{\infty}:=-\hat{A}_{0}-\hat{A}_{1}-\hat{A}_{t_{1}}-\hat{A}_{t_{2}}=\left(\begin{array}{cc}
\theta_{1}^{\infty} & 0 \\
a & \theta_{2}^{\infty}
\end{array}\right) .
$$

После преобразования (37) мы получаем решения

$$
Q_{1}=S_{t_{1}}, \quad Q_{2}=S_{t_{2}}, \quad Q_{3}=S_{1}, \quad Q_{4}=S_{0}
$$

уравнений Шлезингера (5) при $m=4$, при фиксации условий (13) и с постоянными

$$
\theta_{1}=\theta^{t_{1}}, \quad \theta_{2}=\theta^{t_{2}}, \quad \theta_{3}=\theta^{1}, \quad \theta_{4}=\theta^{0} .
$$

Сумма решений (42) задает постоянную матрицу

$$
S_{\infty}=-S_{0}-S_{1}-S_{t_{1}}-S_{t_{2}}=\left(\begin{array}{cc}
\theta_{1}^{\infty} & 0 \\
0 & \theta_{2}^{\infty}
\end{array}\right)
$$

которая совпадает с матрицей (14). Это означает, что в [40] рассмотрены лишь решения системы Шлезингера, которые заменами (12) переводятся в множество решений данных систем в случае матрицы $B_{\infty}(8)$ с отличной от нуля постоянной $k_{\infty}=\theta_{2}^{\infty}-\theta_{1}^{\infty}$.

3.3. При выполнении равенства

$$
\theta_{1}^{\infty}=\theta_{2}^{\infty}
$$

преобразования

$$
S_{\xi}=\left(\begin{array}{cc}
1 & 0 \\
g_{21} & u
\end{array}\right)^{-1} \hat{A}_{\xi}\left(\begin{array}{cc}
1 & 0 \\
g_{21} & u
\end{array}\right)
$$

с функцией $g_{21}$, удовлетворяющей совместной паре соотношений

$$
\left(g_{21}\right)_{t_{k}}^{\prime}-g_{21}(\ln u)_{t_{k}}^{\prime}=-p_{k}\left(\theta^{t_{k}}+p_{k} q_{k}\right), \quad k=i, i+1,
$$

переводят матрицы (38) в решения (42) уравнений Шлезингера (5), сумма которых имеет вид жордановой клетки:

$$
-\sum_{i=1}^{4} Q_{i}(t)=\left(\begin{array}{cc}
\theta_{1}^{\infty} & 0 \\
\nu & \theta_{1}^{\infty}
\end{array}\right) .
$$

Здесь $\nu$ - постоянная, которая посредством формулы $a\left(t_{1}, t_{2}\right)=\nu u\left(t_{1}, t_{2}\right)$ определяет совместное решение уравнений (41) через функцию (40). При этом собственные значения матриц $S_{i}$ суть по-прежнему 0 и постоянные $\theta_{i}$. После переобозначения (43) 
мы получим решения (42) систем Шлезингера (5) при $m=4$ и выполнении равенств (13), сумма которых с учетом сдвигов (12) задается в точности формулой

$$
B_{\infty}=\left(\begin{array}{ll}
0 & 0 \\
\nu & 0
\end{array}\right)
$$

При $a \neq 0$ можно положить $\nu=1$ и получить эти решения с нормировкой (9). Если же $a=0$, то, положив $\nu=0$, получим соответствующие решения системы Шлезингера, редуцирующейся к шестому ОДУ Пенлеве (см. приложение настоящей статьи). Мы не останавливаемся на вопросе совместности равенства $a=0$ с полиномиальной системой Гарнье, поскольку он не принципиален для основных целей нашей работы.

3.4. Полиномиальная гамильтонова система Гарнье (24) первоначально выписана в [35], где она с помощью явного преобразования выведена из системы ГО (19), (20). При этом как постоянные, так и переменные гамильтоновых систем ГО из работ [35] и [38], [40] отличаются друг от друга. В работе [38] указано, что решения данных систем ГО связаны между собой преобразованием Беклунда, однако это преобразование не приведено. Со своей стороны, отметим, что если справедливы неравенства (35) и дополнительные условия 3,4 , то решения полиномиальной системы Гарнье (24) и решения системы ГО (19), (20) связаны простыми формулами:

$$
\begin{aligned}
\lambda_{1}+\lambda_{2}=\frac{t_{1}+t_{2}-\left(1+t_{2}\right) q_{1}-\left(1+t_{1}\right) q_{2}}{1-q_{1}-q_{2}}, \quad \lambda_{1} \lambda_{2}=\frac{t_{1} t_{2}-t_{2} q_{1}-t_{1} q_{2}}{1-q_{1}-q_{2}}, \\
q_{1}=\frac{\left(1-t_{2}\right)\left(\lambda_{1}-t_{1}\right)\left(\lambda_{2}-t_{1}\right)}{\left(t_{1}-t_{2}\right)\left(\lambda_{1}-1\right)\left(\lambda_{2}-1\right)}, \quad q_{2}=-\frac{\left(1-t_{1}\right)\left(\lambda_{1}-t_{2}\right)\left(\lambda_{2}-t_{2}\right)}{\left(t_{1}-t_{2}\right)\left(\lambda_{1}-1\right)\left(\lambda_{2}-1\right)} .
\end{aligned}
$$

Действительно, посчитав элемент “ 12 ” в матрице коэффициентов

$$
\frac{\hat{A}_{0}}{x}+\frac{\hat{A}_{1}}{x-1}+\frac{\hat{A}_{t_{1}}}{x-t_{1}}+\frac{\hat{A}_{t_{2}}}{x-t_{2}}
$$

получим, что он равен

$$
\frac{\left(1-q_{1}-q_{2}\right) x^{2}+\left(-t_{1}-t_{2}+q_{2}\left(1+t_{1}\right)+q_{1}\left(1+t_{2}\right)\right) x+t_{1} t_{2}-q_{1} t_{2}-q_{2} t_{1}}{x(x-1)\left(x-t_{1}\right)\left(x-t_{2}\right)} .
$$

Поэтому равенства (45), (46) следуют из формулы (15) и из того факта, что преобразование подобия (37) с матрицей вида (39) не меняет элемент (48).

С помощью дифференцирования, используя системы (19), (20) и (24), можно проверить, что если ни одна из координат $q_{i}$ и $\lambda_{i}$ не равна тождественно нулю, то справедливы соотношения

$$
\begin{aligned}
p_{1}+\frac{\theta^{t_{1}}}{q_{1}}= & \frac{\left(\lambda_{1}-1\right)\left(\lambda_{2}-1\right)}{\left(t_{1}-1\right)\left(t_{2}-1\right)}\left(\frac{\left(\lambda_{1}-1\right)\left(\lambda_{1}-t_{2}\right) \mu_{1}-\left(\lambda_{2}-1\right)\left(\lambda_{2}-t_{2}\right) \mu_{2}}{\lambda_{1}-\lambda_{2}}-\right. \\
& \left.-\left(\theta^{t_{1}}+\theta^{t_{2}}+\theta^{1}+\theta^{0}+\theta_{2}^{\infty}\right)+\frac{\theta^{0} t_{2}}{\lambda_{1} \lambda_{2}}\right), \\
p_{2}+\frac{\theta^{t_{2}}}{q_{2}}= & \frac{\left(\lambda_{1}-1\right)\left(\lambda_{2}-1\right)}{\left(t_{1}-1\right)\left(t_{2}-1\right)}\left(\frac{\left(\lambda_{1}-1\right)\left(\lambda_{1}-t_{1}\right) \mu_{1}-\left(\lambda_{2}-1\right)\left(\lambda_{2}-t_{1}\right) \mu_{2}}{\lambda_{1}-\lambda_{2}}-\right. \\
& \left.-\left(\theta^{t_{1}}+\theta^{t_{2}}+\theta^{1}+\theta^{0}+\theta_{2}^{\infty}\right)+\frac{\theta^{0} t_{1}}{\lambda_{1} \lambda_{2}}\right) .
\end{aligned}
$$


3.5. Наше замечание 3.4 не решает полностью вопрос о связи системы ГО (19), (20) с полиномиальной системой Гарнье (24) из работ [38], [40].

Рассмотрим, например, редукцию полиномиальной системы Гарнье (24)

$$
q_{i}+q_{i+1}=1
$$

для которой формулы (45) теряют смысл. В случае выполнения равенства

$$
\theta_{1}^{\infty}=\theta_{2}^{\infty}+1
$$

такая редукция существует. В самом деле, сложение уравнений (27) и (28) дает соотношение

$$
\begin{aligned}
t_{i}\left(t_{i}-1\right)\left(q_{i}+q_{i+1}\right)_{t_{i}}^{\prime}=\left(q_{i}+q_{i+1}-1\right)\left(2 p_{i} q_{i}\left(q_{i}-t_{i}\right)+2 p_{i+1} q_{i+1} q_{i}-\right. \\
\left.-\left(\theta^{1}+2 \theta_{2}^{\infty}\right) q_{i}-t_{i} \theta^{t_{i}}\right)+\left(1+\theta^{1}+2 \theta_{2}^{\infty}+\theta^{0}+\theta^{t_{i}}+\theta^{t_{i+1}}\right)\left(t_{i}-1\right) q_{i}
\end{aligned}
$$

которое в предположении, что верно равенство (49), влечет за собой тождество

$$
1+\theta^{1}+2 \theta_{2}^{\infty}+\theta^{0}+\theta^{t_{i}}+\theta^{t_{i+1}}=0 .
$$

Отметим, что при редукции $q_{i}=0, q_{i+1}=1$ система $(24)$ не имеет решений ни при каких значениях $\theta^{\xi}$. Равенства (50) и (52) с учетом соотношения Фукса (26) равносильны.

Правые части уравнений (28) и (31) совпадают. Поэтому из равенства (49) также следует тождество $t_{i}\left(t_{i}-1\right)\left(q_{i}\right)_{t_{i}}^{\prime}+t_{i+1}\left(t_{i+1}-1\right)\left(q_{i}\right)_{t_{i+1}}^{\prime}=0$, означающее, что функция $q_{i}$ зависит от своих аргументов следующим образом:

$$
q_{i}\left(t_{i}, t_{i+1}\right)=q_{i}(\omega), \quad \omega=\frac{t_{i}\left(t_{i+1}-1\right)}{t_{i+1}-t_{i}} .
$$

При выполнении равенств (49) и (50) результаты сложения уравнения (29) с уравнением (33) и уравнения (30) с уравнением (34) совпадают:

$$
t_{i}\left(t_{i}-1\right)\left(p_{i}\right)_{t_{i}}^{\prime}+t_{i+1}\left(t_{i+1}-1\right)\left(p_{i}\right)_{t_{i+1}}^{\prime}=t_{i}\left(t_{i}-1\right)\left(p_{i+1}\right)_{t_{i}}^{\prime}+t_{i+1}\left(t_{i+1}-1\right)\left(p_{i+1}\right)_{t_{i+1}}^{\prime} .
$$

Таким образом, разность $p_{i}-p_{i+1}$ также зависит лишь от независимой переменной $\omega$ :

$$
P\left(t_{i}, t_{i+1}\right)=p_{i}\left(t_{i}, t_{i+1}\right)-p_{i+1}\left(t_{i}, t_{i+1}\right)=P(\omega) .
$$

В силу (53) и (54) уравнение (28) и разность уравнения (29) с уравнением (30) принимают при этом вид гамильтоновой системы:

$$
Q_{\omega}^{\prime}=H_{P}^{\prime}(\omega, Q, P), \quad P_{\omega}^{\prime}=-H_{Q}^{\prime}(\omega, Q, P),
$$

где $Q=q_{i}(\omega)$, а гамильтониан $H$ имеет вид

$$
\begin{aligned}
H= & \frac{1}{\omega(\omega-1)}\left(P^{2} Q(Q-1)(Q-\omega)-P\left(\left(\theta_{1}+2 \theta_{2}^{\infty}\right) Q(Q-1)+\omega \theta^{t_{i}}(Q-1)+\right.\right. \\
& \left.\left.+(\omega-1) \theta^{t_{i+1}} Q\right)+\theta_{2}^{\infty}\left(\theta_{2}^{\infty}+\theta^{1}\right) Q\right) .
\end{aligned}
$$


Если не рассматривать вырожденные решения этой гамильтоновой системы, в которой $Q(\omega)=$ const и $Q(\omega)=\omega$, существующие при дополнительных ограничениях на постоянные $\theta^{\xi}$, то после исключения из системы $(55)$ импульса $P(\omega)$ координата $Q(\omega)$ удовлетворяет общему случаю шестого ОДУ Пенлеве: гамильтониан $H$ совпадает с известным полиномиальным гамильтонианом для этого ОДУ [46]. После нахождения $Q(\omega), P(\omega)$ определение $p_{i}$ и $p_{i+1}$ сводится к решению совместных между собой уравнений Риккати.

При редукции (49) из вида формулы (48) для элемента “12" матрицы (47) видно, что условие 3 из предыдущего раздела не выполнено. Заметим, что равенство (50) противоречит также условию 5. Таким образом, рассмотренные выше конструкции из работ [36], [41] не позволяют привести все решения систем ГО к системам Шлезингера, даже если матрица $B_{\infty}$ для последних имеет вид $(8)$ с $k_{\infty} \neq 0$.

\section{4. "КВАНТОВАНИЯ" ПОЛИНОМИАЛЬНОЙ СИСТЕМЫ ГАРНЬЕ}

Квантовым аналогом формулы связи (46) является замена следующего вида:

$$
\zeta=\frac{\left(1-t_{2}\right)\left(x-t_{1}\right)\left(y-t_{1}\right)}{\left(t_{1}-t_{2}\right)(x-1)(y-1)}, \quad \eta=-\frac{\left(1-t_{1}\right)\left(x-t_{2}\right)\left(y-t_{2}\right)}{\left(t_{1}-t_{2}\right)(x-1)(y-1)} .
$$

Эта замена и явная замена

$$
Y=(x y)^{\alpha}(x-1)^{\beta}(y-1)^{\beta} V
$$

где $\alpha$ и $\beta$ удовлетворяют равенствам

$$
\alpha\left(\alpha+\theta_{4}\right)=0, \quad \lambda=2 \alpha \beta+\left(\theta_{3}+1\right) \alpha+\left(\theta_{4}+1\right) \beta+\beta\left(\beta+\theta_{3}\right)+\left(\theta_{1}+\theta_{2}+1\right)(\alpha+\beta),
$$

переводят решения “квантований” (21), (22) в совместные решения уравнений

$$
\begin{aligned}
t_{1}\left(t_{1}-1\right) V_{t_{1}}^{\prime}=\left[\zeta^{3}-\left(t_{1}+1\right) \zeta^{2}+t_{1} \zeta-\frac{t_{1}\left(t_{1}-1\right) \zeta \eta}{t_{1}-t_{2}}\right] V_{\zeta \zeta}^{\prime \prime}+\left[2 \zeta^{2} \eta+\frac{2 t_{1}\left(t_{2}-1\right) \zeta \eta}{t_{1}-t_{2}}\right] V_{\zeta \eta}^{\prime \prime}+ \\
+\left[\zeta \eta^{2}-\frac{t_{2}\left(t_{1}-1\right) \zeta \eta}{t_{1}-t_{2}}\right] V_{\eta \eta}^{\prime \prime}+\left[-\left(\theta_{3}+2 \beta-1\right) \zeta^{2}+t_{1} \zeta\left(\theta_{2}+\theta_{3}+\theta_{4}+2 \alpha+2 \beta\right)-\right. \\
\quad-\zeta\left(\theta_{1}+\theta_{2}+\theta_{4}+2 \alpha+2\right)+t_{1}\left(\theta_{1}+1\right)-\frac{\left(\theta_{1}+1\right) t_{1}\left(t_{1}-1\right) \eta}{t_{1}-t_{2}}+ \\
\left.+\frac{\left(\theta_{2}+1\right) t_{2}\left(t_{1}-1\right) \zeta}{t_{1}-t_{2}}\right] V_{\zeta}^{\prime}+\left[-\left(\theta_{3}+2 \beta-1\right) \zeta \eta+\frac{\left(\theta_{1}+1\right) t_{1}\left(t_{2}-1\right) \eta}{t_{1}-t_{2}}-\right. \\
\left.\quad-\frac{\left(\theta_{2}+1\right) t_{2}\left(t_{1}-1\right) \zeta}{t_{1}-t_{2}}\right] V_{\eta}^{\prime}+\left[\beta\left(\beta+\theta_{3}\right) \zeta+\left(t_{1}-1\right) \theta_{1} \alpha+t_{1} \theta_{1} \beta\right] V \\
t_{2}\left(t_{2}-1\right) V_{t_{2}}^{\prime}=\left[\eta^{3}-\left(t_{2}+1\right) \eta^{2}+t_{2} \eta+\frac{t_{2}\left(t_{2}-1\right) \zeta \eta}{t_{1}-t_{2}}\right] V_{\eta \eta}^{\prime \prime}+\left[2 \eta^{2} \zeta-\frac{2 t_{2}\left(t_{1}-1\right) \zeta \eta}{t_{1}-t_{2}}\right] V_{\zeta \eta}^{\prime \prime}+ \\
+\left[\eta \zeta^{2}+\frac{t_{1}\left(t_{2}-1\right) \zeta \eta}{t_{1}-t_{2}}\right] V_{\zeta \zeta}^{\prime \prime}+\left[-\left(\theta_{3}+2 \beta-1\right) \eta^{2}+t_{2} \eta\left(\theta_{1}+\theta_{3}+\theta_{4}+2 \alpha+2 \beta\right)-\right. \\
\quad-\eta\left(\theta_{1}+\theta_{2}+\theta_{4}+2 \alpha+2\right)+t_{2}\left(\theta_{2}+1\right)+\frac{\left(\theta_{2}+1\right) t_{2}\left(t_{2}-1\right) \zeta}{t_{1}-t_{2}}-
\end{aligned}
$$




$$
\begin{aligned}
& \left.-\frac{\left(\theta_{1}+1\right) t_{1}\left(t_{2}-1\right) \eta}{t_{1}-t_{2}}\right] V_{\eta}^{\prime}+\left[-\left(\theta_{3}+2 \beta-1\right) \zeta \eta-\frac{\left(\theta_{2}+1\right) t_{2}\left(t_{1}-1\right) \zeta}{t_{1}-t_{2}}+\right. \\
& \left.+\frac{\left(\theta_{1}+1\right) t_{1}\left(t_{2}-1\right) \eta}{t_{1}-t_{2}}\right] V_{\zeta}^{\prime}+\left[\beta\left(\beta+\theta_{3}\right) \eta+\left(t_{2}-1\right) \theta_{2} \alpha+t_{2} \theta_{2} \beta\right] V .
\end{aligned}
$$

В силу операторных соотношений

$$
\frac{\partial}{\partial \zeta} \zeta-\zeta \frac{\partial}{\partial \zeta}=1, \quad \frac{\partial}{\partial \eta} \eta-\eta \frac{\partial}{\partial \eta}=1
$$

эти уравнения можно символически записать как “квантования” (при $\varepsilon=1$ )

$$
\varepsilon \frac{\partial V}{\partial t_{i}}=H_{\mathrm{Gar}, t_{i}}\left(t_{1}, t_{2}, \zeta, \eta,-\varepsilon \frac{\partial}{\partial \zeta},-\varepsilon \frac{\partial}{\partial \eta}\right) V, \quad i=1,2,
$$

определяемые гамильтонианами (25) полиномиальной системы Гарнье.

ЗАМЕЧАНИЕ 6. В силу соотношений (60) уравнения (58), (59) можно записать и в виде $(\varepsilon=1)$

$$
\varepsilon \frac{\partial V}{\partial t_{i}}=H_{\mathrm{Gar}, t_{i}}\left(t_{1}, t_{2}, \zeta, \eta, \varepsilon \frac{\partial}{\partial \zeta}, \varepsilon \frac{\partial}{\partial \eta}\right) V, \quad i=1,2 .
$$

Таким образом, замены (56), (57) и конструкции разделов 2,3 дают решения “квантований” (61) полиномиальной системы Гарнье (24). Эти решения явным образом выписаны через решения совместных уравнений ИДМ (4) с условием (13). При этом коэффициенты данных уравнений ИДМ однозначно выражаются (также явным образом) через множество совместных решений систем (24).

$$
\text { Случай } A_{\infty}=0
$$

ПРИЛОЖЕНИЕ

Рассмотрим случай системы уравнений (4)

$$
\Phi_{x}=A(x) \Phi=\left(\frac{A_{1}}{x-t_{1}}+\frac{A_{2}}{x-t_{2}}+\frac{A_{3}}{x-t_{3}}+\frac{A_{4}}{x-t_{4}}\right) \Phi, \quad \Phi_{t_{i}}=-\frac{A_{i}}{x-t_{i}} \Phi
$$

при $A_{1}+A_{2}+A_{3}+A_{4}=A_{\infty}=0$. В силу последнего равенства

$$
A(x)=\frac{P_{1} x^{2}+P_{2} x+P_{3}}{\left(x-t_{1}\right)\left(x-t_{2}\right)\left(x-t_{3}\right)\left(x-t_{4}\right)} .
$$

Все совместные решения этого случая системы (4) удовлетворяют соотношениям

$$
\sum_{i} \Phi_{t_{i}}=-\Phi_{x}, \quad \sum_{i} t_{i} \Phi_{t_{i}}=-x \Phi_{x}, \quad x^{2} \Phi_{x}+\sum_{i} t_{i}^{2} \Phi_{t_{i}}=P_{1} \Phi
$$

и имеют вид $\Phi=F(r, t, \xi)$, где

$$
r=\frac{x-t_{4}}{x-t_{3}}: \frac{t_{2}-t_{4}}{t_{2}-t_{3}}, \quad t=\frac{t_{1}-t_{4}}{t_{1}-t_{3}}: \frac{t_{2}-t_{4}}{t_{2}-t_{3}}, \quad \xi=\frac{t_{2}-t_{4}}{t_{2}-t_{3}},
$$


а функция $F$ есть совместное решение системы уравнений

$$
\begin{gathered}
F_{r}=\left(\frac{a_{1}(\xi, t)}{r}+\frac{a_{2}(\xi, t)}{r-1}+\frac{a_{3}(\xi, t)}{r-t}\right) F, \quad F_{t}=\left(-\frac{a_{3}(\xi, t)}{r-t}+\frac{a_{3}(\xi, t)}{\xi-t}\right) F, \\
F_{\xi}=Q(\xi, t) F .
\end{gathered}
$$

После калибровочного преобразования $F=g(\xi, t) L$ с такой матрицей $g(\xi, t)$, что $g_{\xi}=Q g$, получаем систему

$$
L_{r}=\left(\frac{b_{1}(t)}{r}+\frac{b_{2}(t)}{r-1}+\frac{b_{3}(t)}{r-t}\right) L, \quad L_{t}=-\frac{b_{3}(t)}{r-t} L
$$

которой удовлетворяет матрица $L=L(r, t)$, уже не зависящая от $\xi$. Известно (см. [47]-[49]), что условие совместности этой системы сводится к шестому ОДУ Пенлеве.

В случае $A_{\infty}=\sum_{i=1}^{m} A_{i}=0$ также верно равенство $\Delta_{\infty}=0$, и к уравнениям (11) добавляется еще одно, которое совместно с ними и с уравнениями (10):

$$
\sum_{i=1}^{m} t_{i}^{2} Y_{t_{i}}^{\prime}+x^{2} Y_{x}^{\prime}+y^{2} Y_{y}^{\prime}=-\left(1+\sum_{i=1}^{m} \frac{\theta_{i}}{2}\right)\left(\theta_{1} t_{1}+\theta_{2} t_{2}+\theta_{3} t_{3}+\theta_{4} t_{4}+x+y\right) Y .
$$

Данное уравнение следует из системы (4). При $m=4$ совместное решение всех рассмотренных уравнений имеет вид $Y=\alpha(t, \xi) G(r, s, t)$, где

$$
s=\frac{y-t_{4}}{y-t_{3}}: \frac{t_{2}-t_{4}}{t_{2}-t_{3}} .
$$

На основе формулы (6) несложно заключить, что $M=\tau(t, \xi) F(s, t, \xi)^{-1} F(r, t, \xi)$. Этот факт согласуется с предположением о том, что вышеупомянутое калибровочное преобразование $F=g(\xi, t) L$ задается матрицей $g(\xi, t)=C L(\xi, t)^{-1}$ : при $C=1$ имеем равенство $M=\tau(t, \xi) L(s, t)^{-1} L(r, t)$. Чтобы уточнить, верно ли это предположение, необходимо исходить из постановки задачи Римана-Гильберта и свести ее к интегральным уравнениям. По всей видимости, формулы Фредгольма показывают, что это предположение справедливо.

\section{Список литературы}

[1] Б. И. Сулейманов, "Гамильтонова структура уравнений Пенлеве и метод изомонодромных деформаций”, Асимптотические свойства решений дифференциальных уравнений, ред. А. М. Ильин, Ин-т матем. Башкирского научного центра УрО АН СССР, Уфа, 1988, 93-102; Б. И. Сулейманов, Дифферени. уравнения, 30:5 (1994), 791-796.

[2] Б. И. Сулейманов, ТМФ, 156:3 (2008), 364-377.

[3] Б. И. Сулейманов, “Квантование некоторых автономных редукций уравнений Пенлеве и старая квантовая теория", Тезисы докладов Международной конференции “Дифференциальные уравнения и смежные воросы”, посвященной 110-й годовщине И. Г. Петровского (Москва, 29 мая - 4 июня 2011 г.), Изд-во МГУ, М., 2011, 356-357.

[4] Б. И. Сулейманов, Уфимск. матем. журн., 4:2 (2012), 127-135.

[5] Б. И. Сулейманов, Функи. анализ и его прил., 48:3 (2014), 52-62.

[6] Д. П. Новиков, ТМФ, 161:2 (2009), 191-203. 
[7] D. P. Novikov, "A monodromy problem and some functions connected with Painleve 6", Painleve Equations and Related Topics, Euler International Mathematical Institute, St.-Petersburg, 2011, 118-121.

[8] Д. П. Новиков, Р. К. Романовский, С. Г. Садовничук, Некоторые новые методы конечнозонного интегрирования солитонных уравнений, Наука, Новосибирск, 2013.

[9] A. Bloemendal, B. Virág, Probab. Theory Related Fields, 156:3-4 (2013), 795-825.

[10] A. Bloemendal, B. Virág, Limits of spiked random matrices II, arXiv: 1109.3704.

[11] A. Zabrodin, A. Zotov, J. Math. Phys., 53:7 (2012), 073507, 19 pp., arXiv: 1107.5672.

[12] A. Zabrodin, A. Zotov, J. Math. Phys., 53:7 (2012), 073508, 19 pp.

[13] A. Zabrodin, A. Zotov, Constr. Approx., 41:3 (2015), 385-423.

[14] А. В. Зотов, А. В. Смирнов, ТМФ, 177:1 (2013), 3-67.

[15] А. М. Левин, М. А. Ольшанецкий, А. В. Зотов, УМН, 69:1(415) (2012), 39-124, arXiv: 1311.4498.

[16] A. M. Levin, M. A. Olshanetsky, A. V. Zotov, JHEP, 10 (2014), 109, 29 pp., arXiv: 1408.6246.

[17] H. Nagoya, J. Math. Phys., 52:8 (2011), 083509, 16 pp.

[18] H. Nagoya, Y. Yamada, Ann. Henri Poincaré, 15:2 (2014), 313-344.

[19] I. Rumanov, Hard edge for beta-ensembles and Painlevé III, arXiv: 1212.5333.

[20] I. Rumanov, J. Math. Phys., 56:1 (2015), 013508, 16 pp., arXiv: 1306.2117.

[21] I. Rumanov, Beta ensembles, quantum Painlevé equations and isomonodromy systems, arXiv: 1408.3847.

[22] I. Rumanov, Painlevé representation of Tracy-Widom $\beta$ distribution for $\beta=6$, arXiv: 1408.3779.

[23] H. Rosengren, Special polynomials related to the supersymmetric eight-vertex model. II. Schrödinger equation, arXiv: 1312.5879.

[24] H. Rosengren, Commun. Math. Phys., 340:3 (2015), 1143-1170, arXiv: 1503.02833.

[25] A. Litvinov, S. Lukyanov, N. Nekrasov, A. Zamolodchikov, JHEP, 7 (2014), 144, 19 pp., arXiv: 1309.4700 .

[26] A. M. Grundland, D. Riglioni, J. Phys. A: Math. Theor., 48:24 (2015), 245201, 15 pp., arXiv: 1405.0968.

[27] R. Conte, I. Dornic, C. R. Math. Acad. Sci. Paris, 352:10 (2014), 803-806.

[28] R. Garnier, Ann. Sci. École Norm. Sup. (3), 29 (1912), 1-126.

[29] А. И. Овсеевич, Докл. РАН, 414:6 (2007), 732-735.

[30] А. И. Овсеевич, Пробл. передачи информ., 44:1 (2008), 59-79.

[31] L. Schlesinger, J. Reine Angew. Math., 141 (1912), 96-145.

[32] А. А. Белавин, А. М. Поляков, А. Б. Замолодчиков, "Бесконечная конформная симметрия в двумерной квантовой теории поля", Инстантоны, струны и конформная теория поля, ред. А. А. Белавин, Физматлит, М., 2002, 224-271; A. A. Belavin, A. M. Polyakov, A. B. Zamolodchikov, Nucl. Phys. B, 241:2 (1984), 333-380.

[33] А. Б. Замолодчиков, В. А. Фатеев, ЯФ, 43:4 (1986), 1031-1044.

[34] M. Sato, T. Miwa, M. Jimbo, Publ. Res. Inst. Math. Sci., 15:1 (1979), 201-278.

[35] H. Kimura, K. Okamoto, J. Math. Pures Appl. (9), 63:1 (1984), 129-146.

[36] K. Okamoto, Isomonodromic deformation and the Painlevé equations, and the Garnier system, Institut de Recherche Mathématique Avancée, Université de Strasbourg, Strasbourg, 1982; J. Fac. Sci. Univ. Tokyo Sect. IA Math., 33:3 (1986), 575-618.

[37] H. Kimura, Ann. Mat. Pura Appl. (4), 155:1 (1989), 25-74.

[38] H. Sakai, Isomonodromic deformation and 4-dimensional Painlevé-type equations, Tech. Report, Univ. Tokyo, Tokyo, 2010. 
[39] H. Kawakami, A. Nakamura, H. Sakai, "Toward a classification of 4-dimensional Painlevé-type equations", Algebraic and Geometric Aspects of Integrable Systems and Random Matrices (Boston, MA, January 6-7, 2012), Contemporary Mathematics, 593, eds. A. Dzhamay, K. Maruno, V. U. Pierce, AMS, Providence, RI, 2013, 143-161.

[40] H. Kawakami, A. Nakamura, H. Sakai, Degeneration scheme of 4-dimensional Painlevé-type equations, arXiv: 1209.3836.

[41] K. Iwasaki, H. Kimura, S. Shimomura, M. Yoshida, "From Gauss to Painlevé. Modern theory of special functions", Aspects of Mathematics, E16, Friedr. Vieweg and Sohn, Braunschweig, 1991.

[42] R. Garnier, Ann. Sci. École Norm. Sup., 43 (1926), 239-252.

[43] М. В. Бабич, УМH, 64:1(385) (2009), 51-134.

[44] A. V. Stoyanovsky, A relation between the Knizhnik-Zamolodchikov and Belavin-PolyakovZamolodchikov systems of partial differential equations, 2000, arXiv: math-ph/0012013.

[45] M. Mazzocco, Int. Math. Res. Not., 12 (2002), 613-646.

[46] K. Okamoto, Proc. Japan Acad. Ser. A Math. Sci., 56:6 (1980), 264-268.

[47] Г. Ф. Федоров, Матем. сб., 11(53):1-2 (1942), 97-120.

[48] А. А. Болибрух, Обратные задачи монодромии в аналитической теории дифберенциалъных уравнений, МЦНМО, М., 2009.

[49] G. Mahoux, "Introduction to the theory of isomonodromic deformations of linear ordinary differential equations with rational coefficients", The Painlevé Property. One Century Later, ed. R. Conte, Springer, New York, 1999, 35-76.

Поступила в редакцию 22.04.2015, после доработки 24.05.2015 